\title{
Usability and decision support systems in emergency management
}

\author{
Mats Danielsson* and Håkan Alm \\ Division of Engineering psychology, Luleå University of Technology, Sweden. Phone: +46920491873
}

\begin{abstract}
The actors in charge of an emergency response are confronted with severe difficulties in coordination and decision making, especially in major accidents. To facilitate coordination, various decision support systems (DSS) integrated in communication systems have been developed. However, many DSS in the rescue service organizations are afflicted with under-use and other usability problems. Drawing on both a literature review and an analysis of recently obtained survey data from rescue personnel concerning usability of common communication system in Swedish emergency organizations, this paper addresses the issue of usability of DSS. It is concluded that the impetus for developing DSS in many cases has been the technological possibilities per se, not taking the decision makers task structure and contextual factors into account. It is argued that priority should be given to functions that provide a visual overview of the event and facilitate storing of the series of decisions made during the response
\end{abstract}

Keywords: decision making, DSS, emergency management, usability

\section{Introduction}

Information and communication technology has ubiquitously been implemented in the Swedish Rescue organizations. Vehicle navigational support, mobile access to property information and hazardous material databases are typical applications that are introduced under the authorities of local counties or county alliances and used as decision support system (DSS) in operative settings. On a national level, a new radio communication network - RAKEL, is currently being rolled out providing for improved voice communication between the rescue services, the police, customs and coast guards.

As a part of a regional R\&D - project - Nordic Safety and Security (NSS) aiming to improve competence and communication within "Blue light" authorities in northern Sweden, a series of usability studies of information and communication systems in local rescue service organizations was conducted. In this part a special emphasis was put on DSS and decision making, with the presumption that ${ }^{*}$

\footnotetext{
Corresponding author: E-mail: matdan@1tu.se
}

development of useful information systems requires knowledge of the decision tasks that they are supposed to support.

Decision making lies at the centre of an emergency response, especially for the person in charge of the whole operation, i. e. the incident commander (IC), but also for other actors on lower levels in the chain of command [1]. This is a common feature among various emergency organizations, be it fire and rescue services, police, coast guard or medical emergency services. A basic problem in emergency management research is that the models developed in the field tend to be specific for the emergency domain studied, which makes generalizations difficult $[2,3]$. Can for instance knowledge about police command and control be transferred to explain judgments when fighting a fire? There is a proliferation of models for various domains making it hard to avoid getting too deeply involved in details of the specific situation differences [10]. This paper purports to provide a framework that is relevant for all emergency contexts, although there is a focus on fire and rescue service organizations

The basic task structure of the response is quite similar between organizations and is oftentimes codified in the various organizations SOP $s$. The 
essence of the structure can be summarized in four stages; 1) Observation and information gathering; 2) Judgment, i. e. situation assessment and seizing up the situation; 3) Decision making, i. e. choosing a course of action among alternatives, and 4) Order, i. e. issuing commands and follow-up of the orders $[2,4]$.

In reality, these stages often overlap and there is of course a feedback-loop in the process as a whole. Most of the DSS implemented in the Swedish Rescue Service organisations are designed for tasks at the operational level ; i. e. adapted to routine responses and to staff at lower levels in the chain of command. Previous studies have shown that many of these systems are afflicted with usability problems in terms of sub-optimal functionality, under-use and low acceptance. The impetus has in many cases been the technological development and not consideration of the actual needs [9].

The aim of this study was to identify the main difficulties perceived by incident commanders related to emergency decision making, thereby contributing to a knowledge basis for future DSS.

\section{Method}

A review of investigation reports from major emergencies in Sweden and their ensuing responses was conducted. These investigations were made by the Swedish Rescue Services Agency, the Swedish Accident Investigation Board and by regional rescue service organizations. Open interviews were then performed with four fire chiefs in a city in northern Sweden with ample experience of acting as IC in minor as well in major emergencies. The interviews took place at the respondents' workplace and were videotaped and subsequently transcribed. A couple of days before the interviews, the respondents were asked to bring to mind their experiences of acting at some non-routine emergency responses.

The review and the interviews centered on the questions:

- In which phase of the response do difficulties typically arise?

- What channels and sources for information are most often used to obtain necessary information?

- What kind of support for his/her main tasks is perceived as currently needed by the IC?

\section{Findings}

- An IC is most active during the initial phases of a response. As early as at the initial alarm call he or she might have to make an overall judgment about the scope and character of the emergency in order to mobilize fitting resources. Whether it is a matter of a minor or a major emergency often becomes apparent on the way to the scene of the event. The ICs first minutes on-scene tend to be decisive for the outcome. It is a matter of actively acquiring relevant information in order to make a correct overall decision. This is perceived as a potentially stressful situation, since the crew often is impatiently waiting for commands while the IC is busy gathering and evaluating information. At the same time the IC has to give priority to the crew's safety, even if it turns out to be a life-saving operation. This leads to a satisficing decision strategy, i.e. the IC chooses an alternative that is just sufficient, even when there might be better alternatives available. When the first decisions are made and the crew is actively working on-scene it will be possible for the IC to attend to other aspects of the response, such as coordinating resources, staff build-up and media relations.

- Sources of information preferred vary heavily depending on type of emergency, but on-scene witnesses are generally seen as very useful, especially if the person has local knowledge of for instance the area or the building affected. There are sometimes flaws in information supply, in the sense that relevant information is unavailable or incomplete. Typical examples mentioned by the respondents are accurate inaccurate drawings and flawed maps of the damage area.

- According to the respondents there is a need for support in the form of written documentation and follow-up of decisions made, especially in major responses. The respondents described difficulties in keeping track of the chain of events and the ensuing decision-order-feedback loop. Such a kind of support could also facilitate post-response investigations and ensuing dissemination of experiences within the organization. Currently, there is a heavy reliance on oral routines within the rescue service organization.

\section{Discussion}

The themes raised by the respondents were in many respects coherent both to experiences from major 
accidents and to general decision making research and to results in decision making research.

Traditional decision making research is mainly related to stages (2) and (3) with focus on peoples use of information processing and choice of an optimal alternative. Most of this research has pitted people's decisions against normative models, identifying typical heuristics and biases. This is, however, not a feasible approach when it comes to emergency management because there are no normative models available. In emergency management, the key decision problem is centred on the relation between the tactical and the strategic aspects of achieving effective command and control of the operation.

Naturalistic Decision Making (NDM) is a research paradigm that is more congenial to the task structure in emergency management. Rather than describing ordinary peoples' inadequacies in making rational decisions, the aim is to explain the proficiency you can find among experts. Why is it that for instance an IC, acting at the fire ground, actually succeeds in making accurate decisions in situations that seem extremely arduous to a layman? The NDM paradigm addresses ill-structured problems, characterized by time pressure and high stakes. It is presupposed that there is a decision-action loop and that there might be conflicting and competing goals due to the unfolding of events but also to organizational norms and constraints $[7,8]$.

The importance of previous experience and the adoption of a satisficing strategy were recurrent themes in the respondents' answers. This is explained by a model within the NDM -paradigm, namely Recognition Primed Decision (RPD) which integrates two cognitive processes; recognition and mental simulation. RPD encompasses comprehension of plausible goals, focusing of the relevant cues in the environment, forming expectations that can function as a check of the correctness of the initial assessment and identifying feasible actions to take. Recognition is at the core of the model. Typical, frequent situations lead to well-rehearsed actions implying that people bring their experience to bear on the decision task. This experience is also decisive for the act of mental simulation. For an IC, the latter might be a question of being able to predict the unfolding of events but also a matter of knowledge of the available resources, for instance the fire brigades capacity. The overall decision strategy becomes satisficing rather than optimizing.

This approach is decidedly intuitive. The cognitive processes are expeditious, parallel and beyond introspection. There is a minimum of time available for going through a series of optional diagnosis or courses of action. It is also promoted by routines and organizational traditions within the rescue service organizations. It has, however, been showed to be infelicitous in major accidents because the IC thus easily becomes stuck to direct minute-operative decisions on-scene, while neglecting long-term planning and staff build-up. Experiences from major responses have shown that the IC and other decision makers can be expected to be well prepared for minor, routine accidents, but face difficulties when the event turns out to be larger and more complex, thus requiring a scaling up of the response and involvement of several organizations [4]. In minor, routine accidents, the IC can rely on previous experiences and his/her thinking can be explained in terms of RPD. A major emergency, however, necessitates another cognitive style where the IC takes a step back and adopts a more analytical strategy taking longer time scales into account. There are different time-scales within which the IC and other emergency decision-makers have to work. These can be seen in different strata, where the lowest stratum is represented by a routine response, such as a dustbin fire and the very highest by international emergency responses initiated by organizational disasters such as the sinking of Estonia. The decision-action-feedback cycle of each stratum can be described and analyzed accordingly. When acting at a high strategic level as in the latter case, the IC cannot rely on previous concrete experience and intuition. Rather, the task requires an analytical mode of thinking.

In contrast with intuitive thinking, analytical processing of information is performed by the decision maker in a serial, rather slow fashion, deliberately adhering to an explicit principle for organizing information provided, while being consciously aware of how they are processed. Most often the end result of an analytical process tends to be correct, but major errors may occur from time to time, - when for instance there are misreading of key information. Thus, confidence in method used tends to be high while confidence in decision made is lower. 
Whether a person adapts an analytical or an intuitive cognitive style is determined by task requirements and contextual factors like time constraints and availability to organizing principles, but also by the way information is displayed [5]. Simultaneously presented, perceptually vivid information is intuition inducing, while serially displayed, abstract information (text, bars, charts, etc.) tends to induce an analytical mode of thinking. This should be taken into account in design of information system aiming to support different decision tasks. There are thus two implications to be drawn from this study for future development of DSS in emergency management.

First, priority should be given to development of systems that provide a visually concrete overview of the event and the damage area during the first phase of the response. This will smooth the progress of the first overall decisions which are perceived as most difficult and also decisive for the outcome of the response. Since the seizing-up of the event is intuitive due to time constraints, perceptually vivid display solutions are to be preferred.

Second, storing of issued commands and unfolding of events must be facilitated by both graphical and textual means. Such DSS are especially valuable in major responses where an analytical approach is called for. This can also contribute to a neutralization of the existing traditions within the rescue service organizations with their heavy reliance of previous experiences and oral communication.

\section{References}

[1] Bergmann, R., (2006). Ambient Intelligence for Decision Making in Fire ServiceOrganizations. University of Trier. Department of Business Information Systems II.

[2] Flin, R. (1996). Sitting in the Hot Seat. New York: J. Wiley.

[3] Flin, R., O'Connor, P, and Crichton, M. (2010). Safety at the sharp end. A guide to non-technical skills. Aldershot: Ashgate.

[4] Fredholm, L. (1996). Decision making patterns in major fire-fighting and rescue operations. Unpublished manuscript: The Swedish War College.

[5] Hammond, K.R.; Hamma, R.M.; Grassia, J., and Pearson, T. (1987). Direct comparison of the efficacy of intuitive and analytical cognition in expert judgment. IEEE Transactions on systems, man and cybernetics, Vol.. SMC-17, No. 5. 753-770.

[6] Kelly, R. T., Morrison, J. G., and Hutchins, S. G., (1996). . Impact of Naturalistic Decision Support on Tactical Situation Awareness. Proceedings of the 40th Annual Meeting of the Human Factors and Ergonomics Society,Philadelphia, PA.

[7] Klein, G. (1993). A Recognition-Primed Decision (RPD) Model of Rapid Decision Making. In G. Klein, J. Orasanu, R. Calderwood and C. Zsambok (Eds), Decision making in action: Models and methods. New Jersey: Ablex Publishing.

[8] Klein, G. (2009). Streetlight and shadows. Cambridge, MA: MIT Press.

[9]Landgren, J. (2007). Information Technology in Emergency and Crisis Response. Doctoral dissertation. University of Gothenburg.

[10]Martin, L., Flin, R., and Skriver, J. (1997). Emergency decision making - a wider decision framework? In R. Flin, E. Salas, M. Strub and L. Martin. (Eds), Decison Making Under Stress: Emerging themes and applications. Ashgate: Aldershot. 